\title{
Applications of a Novel FIB-SIMS Instrument in SIMS Image Depth Profiling
}

\author{
G. McMahon*, J. Nxumalo** and M.W. Phaneuf* \\ * Fibics Inc., 556 Booth St. Suite 200, Ottawa, ON Canada K1A 0G1 \\ ** Semiconductor Insights, 3000 Solandt Rd., Kanata, ON Canada K2K 2X2
}

A relatively new microanalytical technique available to the microscopist is focused ion beam (FIB) microscopy. This instrument's ability to combine high resolution imaging in secondary electron and ion modes, site-specific TEM sample preparation, and semiconductor device modification and repair, make it a truly versatile instrument [1,2]. The area where this tool does not perform well is in chemical microanalysis. Although some general information can be gained from the ion images, especially when oxide phases are involved that can increase secondary ion yields by orders of magnitude, the general solution to this drawback has been the advent of dual electron and ion beam instruments. The general idea behind these instruments is that when microanalysis is required, the operator switches over from the $\mathrm{Ga}^{+}$ion beam to the electron beam and uses EDS to obtain chemical information. The main limitations to this type of instrument are cost, ultimate detection limits and to a certain extent light element analysis.

This paper reports on the capabilities of a unique FIB-SIMS (secondary ion mass spectrometry) system recently installed at Fibics Incorporated. The system is based on the Atomika Instruments 4500 Quadrupole SIMS, fitted with oxygen and cesium floating low energy ion guns capable of producing ion beams with usable currents at energies as low as $250 \mathrm{eV}$, making them ideal for the analysis of ultrashallow junctions. In addition, this system is also fitted with a FEI $25 \mathrm{kV}$ Ga LMIS (liquid metal ion source). To accommodate this gun, the main chamber was placed on an air table and all the turbomolecular pumps were dampened to reduce vibration.

An application of image depth profiling achieved with this instrument is shown in Figure 1, where cross-sectional SIMS images acquired using a $2 \mathrm{kV} \mathrm{Cs}^{+}$beam and a conventional SEM cross-section of a commercial semiconductor device removed from its flip-chip package are shown. Using this method, a visual representation of the dielectric stack is produced. The width of the ion images is $100 \mu \mathrm{m}$. As devices become more complex, it becomes necessary to examine smaller areas with higher lateral resolution than can be readily analyzed with the relatively large spot size of the oxygen and cesium beams. Using the Ga LMIS, it is possible to routinely profile using a $20 \mu \mathrm{m}$ raster with better than $100 \mathrm{~nm}$ lateral resolution, while retaining reasonable depth resolution down to depths of 4 to $5 \mu \mathrm{m}$. An example of a depth profile through this structure using a $20 \mu \mathrm{m}$ raster is shown in Figure $2 \mathrm{a}$, where the various layers are observed. A degradation in depth resolution is observed as one sputters, in this case down $\sim 10 \mu \mathrm{m}$ into the sample. In Figure $2 b$, the $\mathrm{Ga}^{+}$and the $\mathrm{Cs}^{+}$SIMS craters from the analysis areas are shown. The well defined crater walls in the $\mathrm{Ga}^{+}$crater aid in reducing side wall effects during the profile. In fact, a limitation of this technique when applied to insulators is a shadowing of the electron flood gun as one sputters deeper into the sample and the aspect ratio of the crater becomes larger.

References

[1] M.W. Phaneuf, Micron, 30 (1999) 277.

[2] L.A. Gianuzzi et al., Microscopy Research and Technique, 41 (1998) 285. 


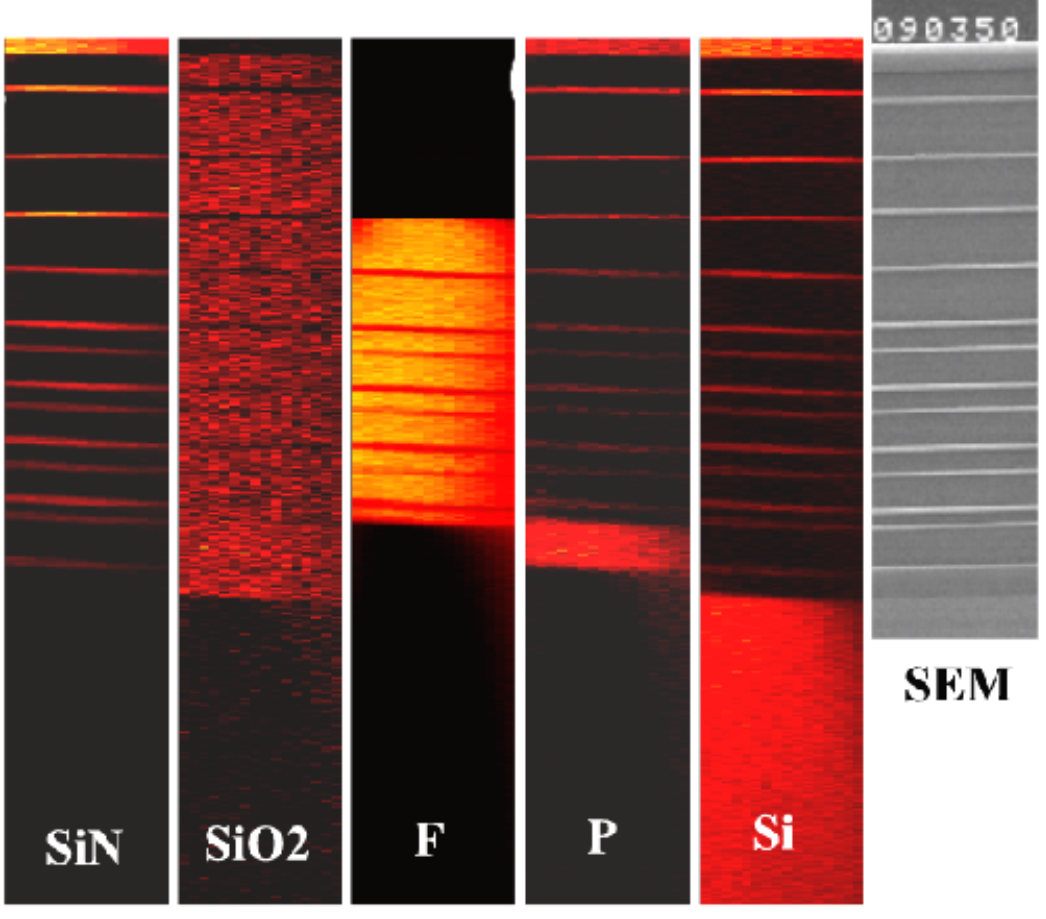

Figure 1: Cross-sectional ion images through the dielectric stack of a semiconductor device. The SEM cross-sectional image is also shown. Width of the SIMS images is $100 \mu \mathrm{m}$.

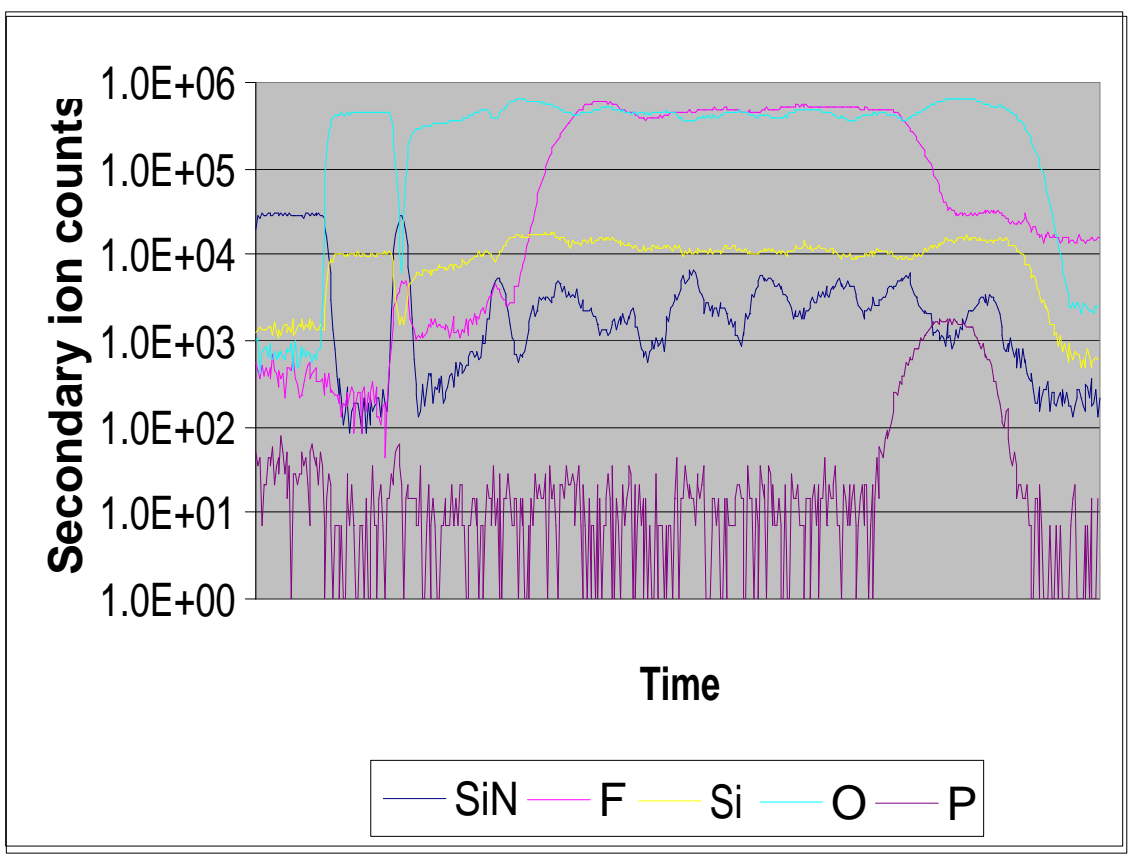

(a)

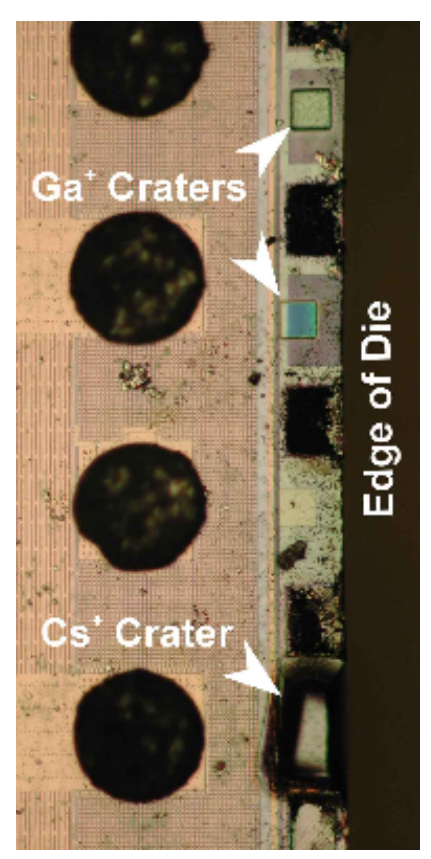

(b)

Figure 2: (a) SIMS depth profile through dielectric stack from (b) $20 \mu \mu \mathrm{m}$ sized crater using a $25 \mathrm{kV} \mathrm{Ga}^{+}$primary ion beam with electron flood charge compensation. 